\title{
Arquitectura religiosa en la Nueva Granada. La catedral de Santa Marta durante el siglo XVIII
}

Religious Architecture in the New Granada. The Cathedral of Santa Marta during the I8th Century

DOI: https://IO.22380/20274688.8I5

\begin{tabular}{c}
\hline Recibido: 25 de febrero del 2019 \\
\hline Aprobado: 21 de junio del 2019 \\
\hline
\end{tabular}

MANUEL GÁMEZ CASAD0*
Universidad de Sevilla, España
mgamez@us.es

\section{R E S U M E N}

El texto que se presenta tiene como objetivo analizar el proceso constructivo de la catedral de Santa Marta durante el siglo XVIII. Si bien se plantearán una serie de antecedentes necesarios para clarificar lo ocurrido en las primeras décadas de aquel siglo, la intervención de arquitectos e ingenieros militares a fines de este permitió concluir el principal edificio religioso de la ciudad. Para clarificar su importancia en el contexto del Nuevo Reino de Granada, partiendo de distintas publicaciones y aportando fuentes primarias, se publicarán nuevos datos y se analizarán distintos planos que contribuirán al conocimiento del edificio.

Palabras clave: ingenieros militares, Nueva Granada, Caribe, arquitectura, siglo XVIII.

* Profesor del Departamento de Historia del Arte de la Universidad de Sevilla. Pertenece al Grupo de Investigación HUM210, laboratorio de arte. Realizó una estancia de investigación en el Instituto de Investigaciones Estéticas de la Universidad Nacional de Colombia, sede Bogotá. Sus publicaciones analizan la labor de los ingenieros militares en la Edad Moderna. https://orcid. org/0000-0002-5539-II04. 


\section{$\begin{array}{llllllll}A & B & S & T & R & A & C & T\end{array}$}

The text presented aims to analyze the construction process of the Cathedral of Santa Marta during the eighteenth century. Although a series of necessary antecedents will be raised to clarify what happened during the 18th century, the intervention of architects and military engineers throughout this century allowed to conclude the main religious building of the city. To clarify its importance in the context of the Nuevo Reino de Granada, starting from different publications and providing primary sources, new data and plans that contribute to the knowledge about the building will be published.

Keywords: military engineers, Nueva Granada, Caribbean, architecture, 18th century.

\section{Introducción}

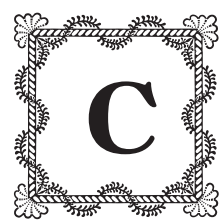

on la fundación de la ciudad de Santa Marta por el viajero sevillano Rodrigo de Bastidas en 1525 y tras la construcción de las primeras casas por los pobladores hispanos en el terreno anteriormente ocupado por los indios mamatocos, la costa meridional del Caribe quedaba parcialmente articulada en beneficio de la Corona española (Bermúdez, Don Rodrigo 63-70) ${ }^{\text {. }}$. Aunque faltaban varios años para que Pedro de Heredia hiciese lo propio en Cartagena de Indias, considerado el principal enclave español del Caribe sur, la privilegiada localización del puerto samario facilitó la llegada de mercaderes que enriquecieron desde temprano las arcas de la gobernación. Se debía proceder entonces a la evangelización de los pueblos circundantes, y el propio Bastidas construyó la primera iglesia, con materiales vernáculos. Esta primera edificación, de la cual se tienen pocas noticias, fue sustituida en 1529 por un nuevo templo de similares características y dimensiones dedicado a Santa Ana, considerado la primera catedral de Santa Marta, categoría que obtuvo mediante una bula promulgada por el papa Clemente VII. En el fraile dominico Tomás de Ortiz recayó la responsabilidad de ejercer como primer obispo de la diócesis (Hernández 7-I8).

Más allá de tales consideraciones, no fue hasta 1549 cuando en tiempos del obispo Martín de Calatayud se construyese la primera catedral de cantería,

I Zambrano y Bernard (18) aportan más información sobre la fundación y población de la bahía de Santa Marta. 
edificio modificado durante el siglo XVII en varias ocasiones debido a los desperfectos ocasionados por ataques piráticos. Debió de ser esta una fábrica modesta, propia de una ciudad portuaria que vivía a la sombra de Cartagena de Indias, pues solo hasta la fundación del Virreinato del Nuevo Reino de Granada en I7I7 fue que se acrecentó su relevancia política y, por consiguiente, la necesidad de levantar un nuevo templo. En realidad, existe un cierto desconocimiento sobre estos primeros proyectos, pues además del estudio de Bermúdez, son escasas las publicaciones que han analizado con rigurosidad los sucesivos planes de reformas emprendidos en la catedral de Santa Marta hasta mediados del siglo XVIII (Bermúdez, Materiales I44-I64). De hecho, desde que Angulo publicase el plano trazado por el ingeniero Juan Cayetano Chacón y Marco Dorta describiese con cierta agudeza el edificio, los investigadores se han limitado a citar estos estudios, sin tratar de aportar novedades documentales o posibles interpretaciones que amplíen el conocimiento sobre su proceso constructivo (Angulo 450; Marco 223-27I). Es el caso del estudio de Hernández Ospino, que si bien parte de un primer análisis documental del edificio, olvida incluir otros aspectos fundamentales relativos a la intervención de varios arquitectos e ingenieros militares durante la segunda mitad del siglo xviII (Hernández 7-8). Más reciente es la publicación de D’Amato, estudio clarificador y detallado acerca de las cuentas y finanzas manejadas durante la construcción de la catedral de Santa Marta. Debe considerársele la más reciente aportación capital sobre el tema. A los comentarios de Corradine y Arango sobre la catedral, se le debe sumar finalmente la guía artística del Caribe colombiano publicada conjuntamente por la Junta de Andalucía y la Universidad del Atlántico, texto que bajo la dirección del profesor Bell recoge algunos de los planos aquí publicados, siendo esta la última aportación relativa al tema.

Además de lo comentado, la historiografía ha obviado la importancia de la catedral dentro del agitado contexto artístico propio de la historia caribeña del siglo Xviri. Por todo ello, será ocupación del presente texto relatar el proceso de reconstrucción de la catedral de Santa Marta tras su destrucción a causa del fatídico terremoto de $\mathbf{1 7 5 2}$. Precisamente en ese mismo año se fundó la Real Academia de Bellas Artes de San Fernando de Madrid, institución que propició un cambio determinante en los modelos estéticos para las propuestas de los ingenieros y arquitectos que trabajaron en las Indias². Es más, en 1777 se le otorgó 
la potestad para evaluar los proyectos de obras públicas, civiles y religiosas, de todo el territorio controlado por la Corona, incluyendo los virreinatos indianos, e influyó decisivamente en el devenir de la arquitectura neogranadina de las últimas décadas del siglo XVIII (Ruiz). Ello permitirá comprobar el grado de integración de las experiencias clasicistas en el contexto virreinal, al relacionar lo construido con la aparición de nuevas tendencias arquitectónicas surgidas en ese momento. Con tal fin, se estudiará el caso particular de la catedral de Santa Marta, edificio diseñado por ingenieros militares, iniciando una línea de estudio que podría ser aplicada a otros casos dentro de las capitales neogranadinas.

\section{La catedral de Santa Marta en el contexto del Nuevo Reino de Granada}

El Virreinato del Nuevo Reino de Granada fue la tercera de las divisiones políticas, territoriales y administrativas que la Corona española realizó en el continente americano desde su descubrimiento. Iniciada esta reforma en $17 \mathrm{I} 7$, inauguraba la centuria más compleja de la historia indiana, pues tras los conflictos de la guerra de Sucesión se encadenaron otros que sumieron a la monarquía en una decadencia inusitada. Al incrementar este declive contribuyeron las potencias europeas presentes en el teatro bélico caribeño, caso de Inglaterra y Holanda, que organizaron continuos ataques contra los dominios españoles. Además, entre dichos países y los indios locales surgió un comercio ilegal, difícilmente controlable por las autoridades españolas, que debilitó la estructura administrativa del reino y situó en el puerto de Santa Marta el principal foco de acción contrabandista de la zona (M. Restrepo 245). De hecho, hasta las políticas implantadas por el ingeniero militar y gobernador de Santa Marta, Antonio de Narváez en $\mathbf{1 7 7 8}$, no se tomaron las primeras medidas políticas en contra de esta lacra, resultado de los nuevos principios ilustrados surgidos en este contexto (Gámez, "De ingeniero voluntario" 239).

También durante el gobierno de Narváez se implementaron una serie de reformas con la intención de paliar la despoblación de Santa Marta, problema considerado capital para el desarrollo económico de la ciudad y la consolidación de su red comercial. A mediados del siglo Xviı la desigualdad poblacional era todavía latente, pues numerosas tribus no estaban controladas o no vivían en poblaciones regulares. De los 60.000 habitantes que se contaban en Santa Marta en torno a I770, más de la mitad pertenecían a las etnias de los chimilas, 
los motilones y los guajiros, y constituían una población alejada del control político y las normas sociales impuestas por la gobernación (Borrego 74). La hostilidad de estas tribus impedía a los naturales, que se asentaban al rededor del Magdalena buscando las zonas más fértiles en beneficio de sus cultivos, trabajar en los campos.

Asimismo, el declive de la autoridad religiosa en la provincia animó el crecimiento de otras creencias paganas. Precisamente para frenar estas actuaciones se implantó un sistema de encomienda, creado con la intención tanto de recompensar los méritos de los conquistadores, como de lograr la cristianización de los indígenas. Si bien es cierto que durante el siglo Xvin la encomienda experimentó una importante crisis en la Nueva Granada, esta medida administrativa resultó fundamental en poblaciones hostiles al control político, las cuales quedaron sujetas al mandato de un beneficiario (Molino 5-6). Con la misma intención doctrinera se propuso la sustitución de la vieja catedral por un nuevo edificio de mayores dimensiones, que propagase la grandeza del nuevo virreinato y de la autoridad eclesiástica, que se debía imponer ante la población local. Sin duda, en la confirmación del poder religioso ante la población indígena de Santa Marta subyace la reconstrucción de la catedral, lo que explicaría las amplias dimensiones del templo, la sucesión de los planteamientos presentados y el interés de la gobernación por finalizar el edificio. Este último iba a convertirse en un ejemplo evidente de arquitectura propagandística en la Nueva Granada. Precisamente la resistencia mostrada por los indígenas, la aplicación de la estructura encomendera en Santa Marta y el incremento masivo del contrabando derivaron en una escasez de mano de obra local que produciría una demora en la culminación del nuevo templo, como se estudiará más adelante.

A pesar de estas intenciones, parece que con la fundación del Nuevo Reino, la Corona no destinó los fondos necesarios para las labores de conservación requeridas por los edificios religiosos de la Audiencia de Santa Fe, de lo que es prueba el deterioro que presentaba la catedral de Santa Marta en los años centrales del siglo xviri. Es más, en la descripción que el obispo Juan Nieto Polo realizó del edificio en 1749 calificó su estado como ruinoso, pues el tabernáculo del altar mayor estaba destruido y existía un palpable riesgo de derrumbe. Incluso, tanto Nieto como su sucesor en el cargo, José Javier Arauz, culparon al cabildo civil de ser el responsable del abandono del templo, ya que durante el tiempo de sede vacante recaía en la institución municipal el cuidado de la catedral (AGI, $S F$, sign. II80, "Carta del obispo de Santa Marta José Javier Arauz", I9 de abril de I750). Es evidente que los fondos destinados a su conservación se 
empleaban en otros asuntos, circunstancia denunciada por los sucesivos obispos de la diócesis sin que tuviesen respuesta por parte de las autoridades virreinales.

Aunque las relaciones entre el obispo Arauz y el virrey Solís se fueron consolidando paulatinamente, es indudable que las prioridades constructivas en Santa Marta eran otras. Aún a mediados de siglo se podían ver las carencias defensivas que la ciudad había ido acumulando desde la guerra de Sucesión. A este malestar contribuían las revueltas protagonizadas por los indios, quienes pretendían cortar las comunicaciones entre el puerto samario y los otros mercados cercanos ${ }^{3}$. Igualmente, las consecuencias producidas por los ataques comandados por el vicealmirante Vernon en las costas del reino mermaron las posibilidades de defensa y prosperidad del territorio. A todo ello se le debía sumar la inestabilidad política e institucional del virreinato, cuya fundación no terminó de consolidarse hasta la llegada del virrey Sebastián de Eslava en 1739.

Es bien sabido que el arribo de Eslava supuso un importante cambio en el panorama político del virreinato. A su labor se atribuye la obtención de beneficios económicos generados por la acumulación de esclavos, la explotación de las minas de oro del Chocó y de piedras preciosas en la meseta de Cundinamarca, el comercio en el puerto de Cartagena y la implantación de las rentas de aguardiente y tabaco. Con la explotación de estas actividades y superadas las dificultades propias de un reino recién fundado, se inició la etapa de mayor esplendor para las audiencias neogranadinas. Precisamente coincidiendo con el inicio del reinado de Carlos III y como consecuencia de la firma de los pactos de familia, se incluyó, en 1776, a los puertos de Riohacha y Santa Marta en el régimen de las Antillas, medida que facilitó el comercio directo con los mercados franceses y generó una extraordinaria riqueza en los puertos secundarios (Navarro 96-98).

Con todo ello, el virreinato estaba plenamente consolidado dentro del panorama político indiano, y se inició una etapa fecunda de la que es prueba la construcción de algunos edificios religiosos y civiles y, sobre todo, los principales sistemas defensivos del Nuevo Reino. Además del remozamiento de viejas iglesias mediante la construcción de novedosas fachadas, de las que son pruebas la dedicada a san Francisco en El Tocuyo o la parroquia de El Pao,

3 Numerosas fueron las medidas impuestas por los Borbones para repeler las incesantes revueltas protagonizadas por los guajiros, entre las cuales destacaron la construcción de fortificaciones y la organización de expediciones comandadas por ingenieros militares. Al respecto, véase Gámez, "La pacificación" (373-386). 
ambas situadas en Venezuela, en la segunda mitad del siglo Xvin también se edificaron imponentes iglesias de nueva planta. Es más, la renovación de la catedral de Panamá por el ingeniero Manuel Hernández, la construcción de la iglesia del convento de San Francisco de Popayán bajo la dirección del arquitecto Antonio García, o la conclusión de la catedral de Santa Fe de Antioquia por el arquitecto santafereño José Ortiz, son buena prueba del agitado contexto artístico en las audiencias neogranadinas. Similares características formales presentan tanto las construcciones citadas como otras pertenecientes al mismo territorio, imperando una arquitectura ordenada, de escasa decoración y con un gusto notorio por el clasicismo.

Estas formas deben relacionarse con las últimas novedades estéticas surgidas tras la fundación de instituciones formativas como las academias de matemáticas de Barcelona, Orán o Ceuta, donde estudiaron los ingenieros que viajaron a la Nueva Granada para construir fortificaciones, obras públicas y edificios religiosos. Además de ciertos modelos vitruvianos y vignolescos, en estos centros conocieron algunos tratados capitales para el estudio de la arquitectura civil y religiosa, establecidos por Mateo Calabro y Pedro Lucuce como mejora del plan formativo instaurado para los cadetes. Entre otros, los textos de Prósperi, Tosca o Bélidor asentaron la base teórica de los proyectos constructivos derivados de dichas academias, cuyos diseños fueron trasladados a América por los ingenieros (Galindo). Como resultado de esta traslación de modelos, cabe citar la construcción de la puerta del puente de Cartagena de Indias por Herrera y Sotomayor, basándose en los modelos clásicos propios de la tradición europea, o el diseńo del hospital de San Lázaro de la misma ciudad por Antonio de Arévalo, siguiendo los esquemas de Bélidor. En la misma línea destaca el proyecto de Juan Ximénez Donoso para la fachada del palacio virreinal de Bogotá, cuyo frente apilastrado confirma la utilización de los tratados europeos como fuentes para la arquitectura americana durante el siglo xviri. Asimismo, la fundación de varias academias de ingenieros en diferentes ciudades, como la organizada en 1725 por Herrera y Sotomayor en Cartagena, confirma la asimilación de estos programas formativos en la Nueva Granada. Ello demuestra la traslación de ideas, modelos y teorías entre ambas orillas del Atlántico y el incentivo a la renovación arquitectónica durante el siglo XVIII (Marchena 23-28). Por todo lo anterior, cabe pensar que la influencia de la Academia de Matemáticas de Barcelona fue decisiva en las actuaciones de los ingenieros en América.

Dentro del amplio contexto descrito se debe encuadrar la construcción de la catedral de Santa Marta, edificio singular en su género y único en el 
ámbito caribeño. La construcción del templo, a propuesta de la gobernación, tenía como objetivo principal la consolidación de la política borbónica en un área conformada por realidades sociales dispares. La intención era reafirmar el poder religioso frente a otras creencias, circunstancia que explica la implicación de la gobernación en la finalización del edificio.

\section{Las intervenciones del ingeniero Manuel Hernández y del arquitecto Diego de Rueda}

Tras superar los primeros envites políticos propios de un virreinato de reciente fundación, las audiencias neogranadinas pudieron prosperar gracias al incremento de los beneficios económicos obtenidos durante la segunda mitad del siglo XviII. Ello posibilitó reiniciar el ambicioso proyecto de remodelación de la catedral de Santa Marta, cuya infortunada situación se acentuó tras una serie de terremotos acaecidos entre 1749 y 1752 . Estos seísmos destruyeron parte de la techumbre, varios arcos formeros y algunos pilares, lo que obligó a trasladar la celebración de los oficios al cercano convento de San Francisco ante el riesgo de derrumbe. En abril de 1752, el obispo Arauz solicitó al teniente coronel y gobernador de Santa Marta, Antonio de Alcalá Galiano que enviase al ingeniero militar que desde Cartagena de Indias había sido comisionado por el virrey para inspeccionar las fortificaciones del litoral caribeño, también afectadas por los sucesivos seísmos. El ingeniero en cuestión era Manuel Hernández, militar experimentado en el diseño de fortificaciones y de edificios civiles, pues había trabajado en el diseño de varios embarcaderos en las Canarias y un año antes había dirigido la rehabilitación de la aduana de Portobelo, destruida tras el bombardeo de Kinghills (Gámez, "Ingenieros militares" I25-I38)4.

Hernández era reconocido en América como uno de los ingenieros más destacados, por su exquisita formación en la Academia de Matemáticas de Barcelona y su extraordinaria experiencia al frente de diversos proyectos urbanísticos, primero en Cartagena y posteriormente en Panamá y Portobelo. Sin embargo, lo que realmente condicionó su llegada a Santa Marta fue su extraordinaria

4 Sobre dicho ataque, véase Castillero (450-454). Para conocer más sobre la producción del ingeniero militar Manuel Hernández, consúltese Capel et al. (227-228). 
labor al frente de la reconstrucción de la catedral de Panamá en I749, donde además de modificar la planta del viejo edificio, sustituyó los antiguos pilares por otros de menor grosor que aumentaban la visibilidad del altar mayor. El éxito de estos trabajos le sirvió de aval para trabajar en Santa Marta, pasando de inmediato a inspeccionar la catedral a fines de abril de 1572. No obstante, pronto reconoció que era incapaz de remediar el lamentable estado en el que se encontraba la edificación, por lo abierto de sus arcos, muros y puertas. Además, la torre estaba inclinada y presentaba numerosas grietas, por lo que recomendó a la gobernación que se construyese una nueva catedral para evitar desplomes y un gasto excesivo de dinero.

Para iniciar la rehabilitación del edificio, desde la Corte se le asignó al gobernador Antonio de Alcalá la cantidad de 6.000 pesos y los dos reales novenos del diezmo de la provincia durante diez años. Es evidente que tal cantidad era suficiente para construir lo proyectado por Hernández. Sin embargo, el obispado se gastó la asignación inicial en el adecentamiento superficial del edificio, comprando un juego de ornamentos de damasco guarnecidos en oro y plata, una nueva escultura de Santa Marta traída desde Quito e incluso una nueva custodia de plata sobre dorada rematada por una cruz de oro enjoyada con amatistas (AGI, SF, sign. II88, "Carta del gobernador Antonio de Alcalá", I4 de octubre de 1752). Como la adquisición de estas piezas permitió adecentar la ruinosa catedral, el gobernador destinó los fondos necesarios para la reconstrucción de otros templos menores situados en la provincia con el fin de controlar a los pueblos nativos. Entre estos, los construidos en el Morro, el Yucal o las sabanas de San Juan Bautista requerían un mayor control y adoctrinamiento. El mismo caso ocurría con los indios de Ocaña, especialmente violentos contra la presencia española en la zona. Así, estas pequeñas construcciones religiosas se utilizaban para vigilar a las belicosas poblaciones locales.

No eran ajenos en la Corte a las noticias que negaban cualquier posibilidad de rehabilitar la vieja catedral. Para evitar que el proyecto quedase en el olvido de los gobernadores, la Corte obligó a la construcción de un nuevo edificio mediante una real cédula promulgada el 3I de octubre de I753 (AGNC, MM, leg. II7, ff. 8 r. y v.). Se debe considerar que esta medida era consecuencia del rápido crecimiento económico experimentado por la ciudad desde los albores del siglo Xviri. Al igual que se invirtió en la construcción de nuevas fortificaciones que defendiesen el puerto, el cual servía como punto intermedio para los comerciantes que partían con rumbo a la Península desde Cartagena, también se sufragó una nueva catedral acorde a la importancia adquirida por la diócesis 
samaria durante el siglo XviIs'. El arquitecto Diego de Rueda, elegido como maestro mayor de obras por orden del virrey José Solís Folch de Cardona, se ocupó de la delineación de los planos de la nueva iglesia. Ciertamente, son escasas las noticias que aporten información acerca de la trayectoria de Rueda. Algunos reconocimientos en las fortificaciones de Cartagena son sus únicos trabajos documentados. Es más, aunque Bermúdez le atribuya el grado de ingeniero, su nombre no se ha localizado en las listas de egresados de las academias de matemáticas españolas, lo que demostraría que su formación estuvo vinculada a otros ámbitos arquitectónicos alejados de la milicia. Por otro lado, la falta de calidad de los dibujos conservados demuestran la escasa preparación de Rueda en esta materia, en contra de la precisión y el hábil dominio que presentan los realizados por los ingenieros.

Tras su elección como nuevo arquitecto de la catedral de Santa Marta, Rueda se encargó de cimentar el nuevo edificio, cuyas dimensiones eran $25 \mathrm{~m}$ de ancho y $43 \mathrm{~m}$ de largo. Para ello, propuso reutilizar los materiales del viejo, y además envió al obispado un plano de su proyecto, que se ha podido fechar en marzo de 1757 (figura I) (AGI, SF, sign. II88, "Carta del arquitecto Diego de Rueda”, 5 de marzo de 1757$)^{6}$. En el dibujo, firmado por Rueda, se representa la planta de la nueva catedral, compuesta por tres naves de cinco tramos divididos por pilares cuadrangulares, mientras que los situados en el crucero son cruciformes. Son precisamente estos soportes los que marcan el transepto, pues como era habitual en la arquitectura neogranadina de la segunda mitad del siglo XVIII, este no se acusa en planta. La cabecera se resuelve con una capilla mayor con testero plano, flanqueada por el sagrario en el lado del evangelio y la sacristía en el opuesto. Además, Rueda incluyó un coro en el centro del templo, como era preceptivo en las catedrales españolas. Asimismo, sugirió la construcción de un baptisterio bajo la torre de la fachada, adosada al exterior del muro de la epístola. Aprovechando el saliente generado por el campanario, se dispuso, en forma de salones alargados, algunos corredores a modo de claustro, adoptando una solución ya utilizada en los primeros proyectos de la catedral de Panamá por el

5 El principal estudio que ha analizado el proceso constructivo de las fortificaciones de Santa Marta se debe a Zapatero.

6 El plano ya fue reproducido en Bell (94). Por otra parte, Torres Lanzas lo fechó en 1647, fecha que no corresponde con los nuevos datos localizados sobre la intervención de Rueda en la catedral (Torres 4I). 
maestro Pedro de Torres. El acceso a la iglesia se realizaría por las tres portadas abiertas en la fachada o las dos ideadas en los ejes de las naves laterales.

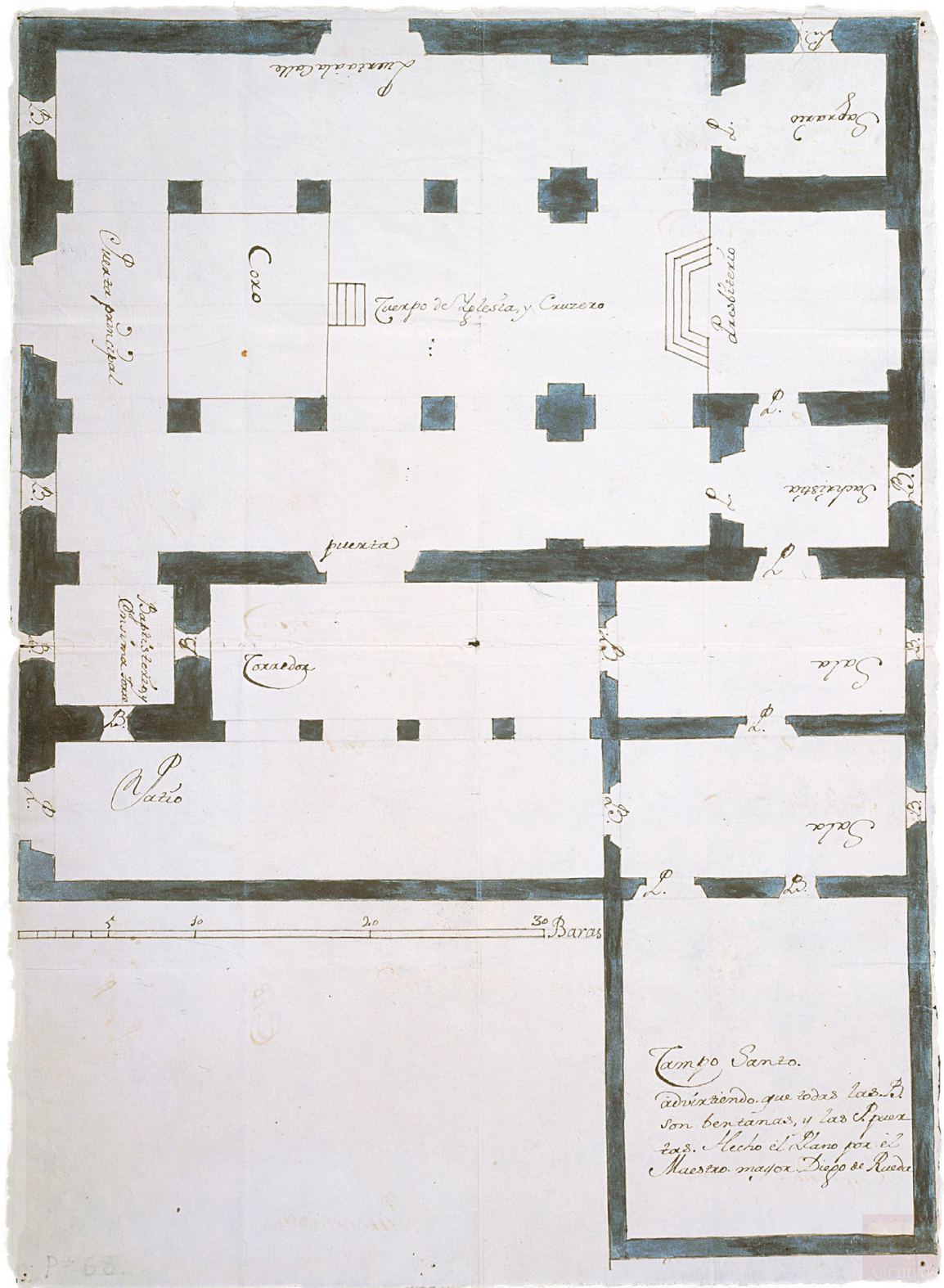

$\rightarrow$ FIGURA I.

Diego de Rueda. Proyecto para la catedral de Santa Marta, I757

Fuente: AGI, MP, P, 68. 
Al parecer, la ruina del viejo templo era absoluta, tanto que el propio Rueda advirtió en varias ocasiones de la necesidad de construir este nuevo edificio ante el riesgo de derrumbe. De hecho, prueba del lamentable estado en que se encontraba fue la caída de la techumbre del coro durante la celebración de las fiestas del arcángel san Miguel, lo que obligó a trasladar los enseres, imágenes y demás ornamentos sagrados al cercano hospicio de San Francisco, y se procedió a desmontar la cubierta debido el riesgo ocasionado (AGI, $S F$, sign. II88, "Carta del obispo de Santa Marta al gobernador", I3 de marzo de 1757)7.

\section{El ingeniero militar Juan Cayetano Chacón y el proyecto definitivo de la catedral de Santa Marta}

Aunque el proyecto de Rueda parecía en principio convincente, pronto cayó en el olvido como consecuencia de la indecisión de las autoridades locales. Precisamente para evitar tal desenlace, el 23 de enero de 1766, el propio virrey Pedro Messía de la Cerda envió desde Cartagena al ingeniero militar Juan Cayetano Chacón como encargado de dirigir el diseño y los trabajos en el nuevo templo (AGNC, $M M$, leg. I22, ff. 797-802). Tras el paso de Hernández por Santa Marta, nuevamente se volvía a confiar en un ingeniero militar de cierta experiencia para comandar las obras, lo que demuestra el papel protagonista de estos profesionales en los contextos arquitectónicos del Nuevo Reino. Aunque las noticias que se han publicado sobre Chacón sean escasas, sí parece obvio que debió de estar integrado al bullicioso y prolífico ambiente ingenieril cartagenero de las décadas centrales del siglo XVIII, precisamente cuando se estaban realizando las reformas de las principales fortificaciones por militares tan representativos como Lorenzo de Solís, Antonio de Arévalo o Juan Bautista Mac-Evan.

Antes del encargo definitivo, se conoce que Chacón había colaborado con el Cabildo de Santa Marta mediante la supervisión de distintos planos y proyectos de reformas encargados por las autoridades a varios arquitectos. Es más, se han encontrado noticias acerca de unos planos trazados por un desconocido arquitecto llamado Diego González, remitidos a Cartagena de Indias para que

7 Para más información puede verse Bermúdez, Materiales (I43).

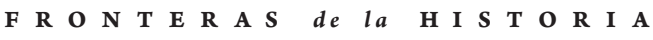


fuesen revisados por Chacón. A la labor de este arquitecto deben corresponder dos bocetos, reproducidos junto a estas líneas, en los que se muestra una sección de las naves del nuevo edificio cubiertas por bóvedas de cañón (figuras 2 y 3). A este primer plano le acompaña otro en el que se representó de nuevo la planta de la catedral, que parece repetir el modelo de Rueda, y una vista de la portada principal y del campanario. El esquematismo y la torpeza del dibujo no impiden vislumbrar que la intención de González era trazar una portada flanqueada por medias columnas dórico-toscanas, las cuales resultan desproporcionadas y desmedidas en relación con el conjunto.

Fue ocupación de Juan Cayetano Chacón reconocer el proyecto de González, por medio de un informe fechado el 3I de octubre de 1765. En dicho escrito mencionó la escasa calidad del proyecto, lo que le hacía pensar que González no fuese más que un maestro albañil. Además, criticó las medidas de la planta, que no cumplían con la proporción dupla sexquialtera utilizada en muchas de las catedrales americanas, y la falta de coherencia del orden columnario, al haber confundido varios frisos, basas y capiteles. Interesante resulta la apreciación de Chacón acerca de los arcos torales, al considerar fundamental para el correcto diseño que estuviesen situados cerca de los estribos exteriores, con el fin de soportar el peso de las bóvedas. Con ello reforzaría la base de la cúpula y eliminaría cualquier riesgo de derrumbe ante los continuos terremotos (AGNC, $M M$, leg. I22, ff. 798-800). En definitiva, los argumentos de Chacón sirvieron para desestimar el proyecto de Diego González, lo cual obligó a la gobernación a reconocer la inexistencia de arquitectos capaces de dirigir la obra. Tal afirmación forzaba a las autoridades a requerir la llegada de un personal experimentado, por lo que se solicitó de inmediato la llegada del ingeniero Juan Cayetano Chacón a las obras de la catedral de Santa Marta ${ }^{8}$.

Las críticas de Chacón al proyecto de González expuestas ejemplifican con precisión el debate suscitado en América sobre la relación entre arquitectos e ingenieros durante el siglo XviII. Esta disyuntiva era el resultado de las distintas políticas de formación planteadas para unos y otros. Si los primeros aún partían de un aprendizaje gremial, que podía resultar retardatario en los contextos surgidos a mediados de la centuria, los ingenieros se habían adaptado a las nuevas exigencias formales mediante el estudio de las matemáticas, la geometría y otras ciencias que mejoraron la práctica de estos militares. Los ingenieros se 


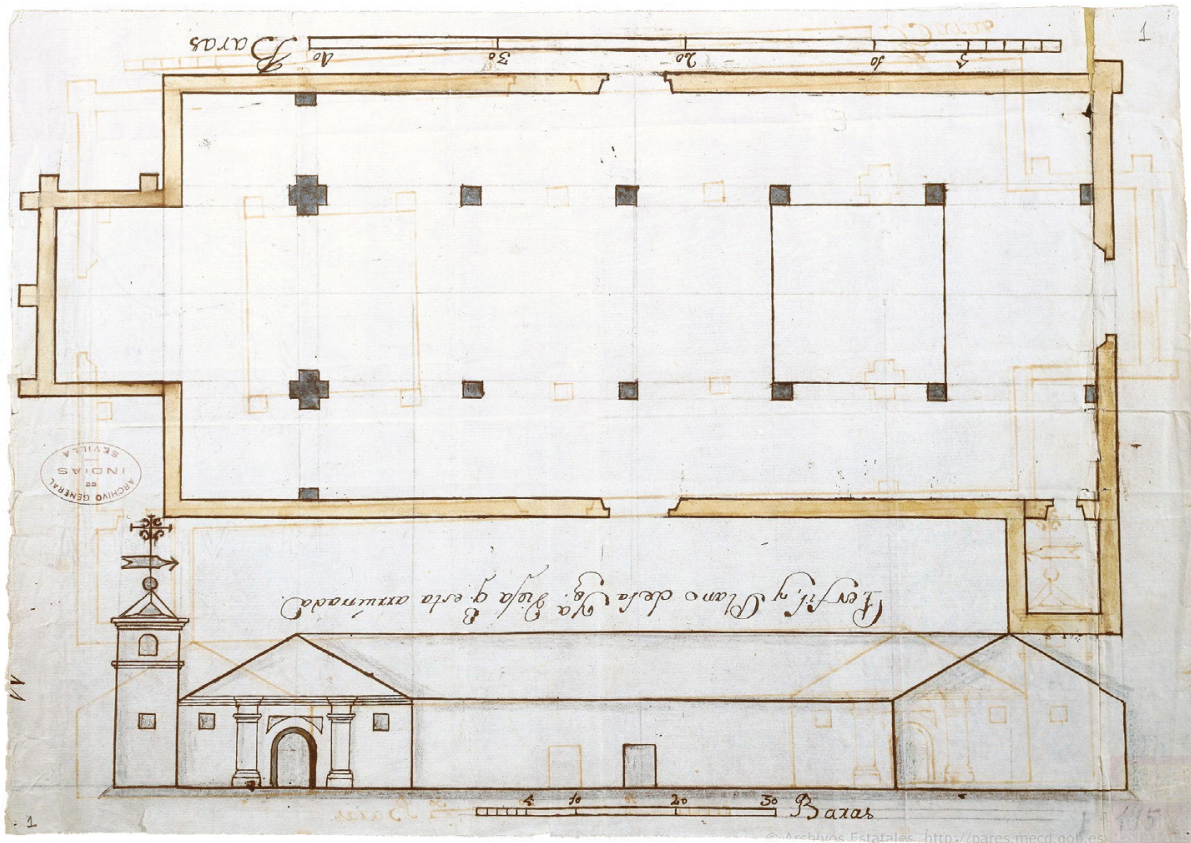

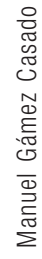

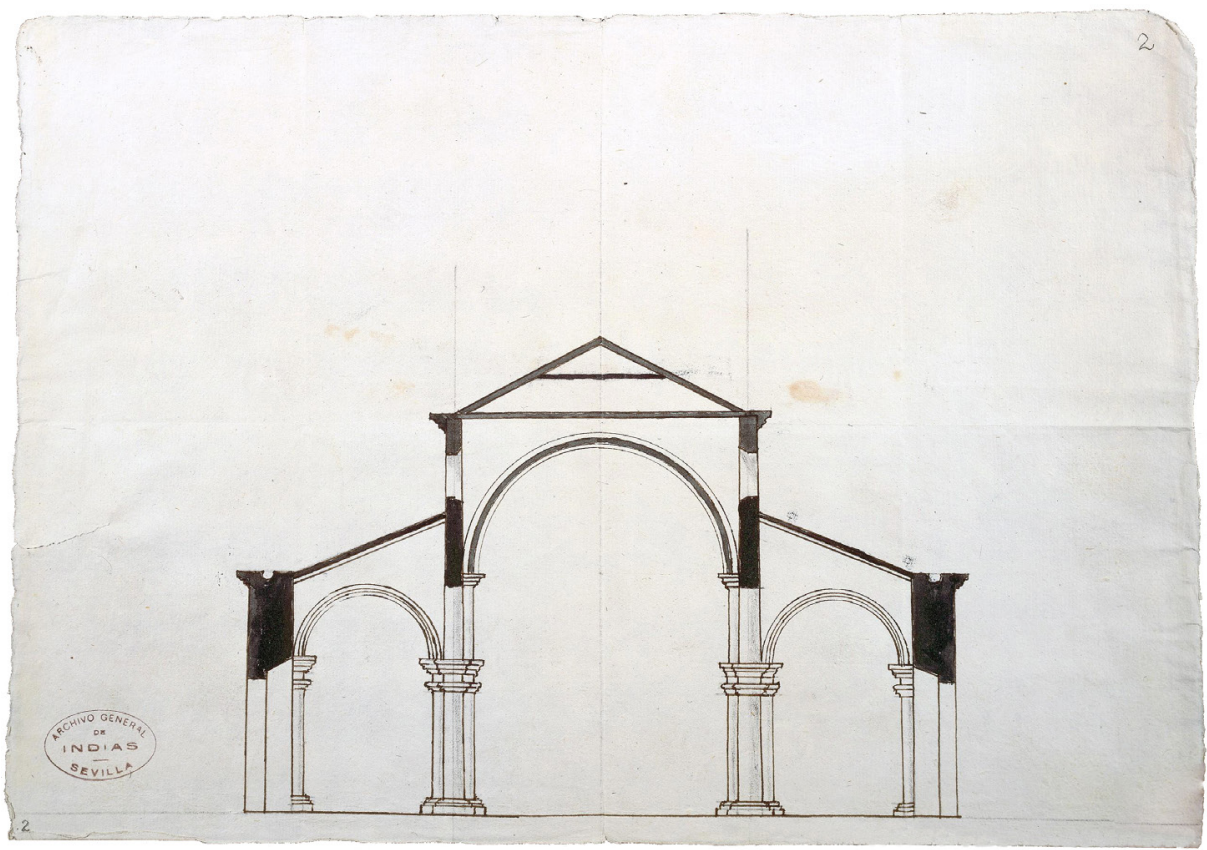

$\rightarrow$ FIGURAS 2 Y 3.

Diego González. Proyecto para la catedral de Santa Marta, I765

Fuente: AGI, MP, P, I75.

$\begin{array}{lllllllllllllllllll}\mathbf{F} & \mathbf{R} & \mathbf{O} & \mathbf{N} & \mathbf{T} & \mathbf{E} & \mathbf{R} & \mathbf{A} & \mathbf{S} & d e & l a & \text { H } & \mathbf{I} & \mathbf{S} & \mathbf{T} & \mathbf{O} & \mathbf{R} & \mathbf{I} & \mathbf{A}\end{array}$ 
convertían en un personal garante para la dirección de los proyectos más complejos emprendidos por la Corona a un lado y otro del Atlántico, relegando a los arquitectos a un segundo plano en las principales plazas indianas. Estas diferencias, que requerirían un estudio monográfico en relación con América, se vieron reflejadas en las obras de la catedral de Santa Marta, pues Chacón se convertiría a la postre en el responsable de la finalización del conjunto.

Superadas estas primeras revisiones y aceptada la propuesta de construir una nueva catedral, Messía le encargó a Chacón la realización de un primer diseño, concluido por el ingeniero el 23 de julio de 1766 , fecha en la que se envió a la Corte (AGI, $S F$, sign. II88, "Carta del virrey Pedro Messía de la Cerda”, i2 de septiembre de I766). Aunque no existe constancia de este primer boceto, sí se ha localizado la relación de gastos que el ingeniero propuso a las autoridades, la cual asciende a un total de 59.859 pesos y contempla el adecentamiento del terreno, la compra de herramientas y el pago de los jornales a los operarios responsables de la construcción del nuevo edificio (AGI, $S F$, sign. II88, "Real Decreto", 5 de octubre de 1766). Además, en relación con este primer dibujo se han hallado diversas cartas en las que el ingeniero detalla que lo hizo en Cartagena, sin tener un conocimiento preciso de la consistencia del terreno. A ello lo obligaron las dimensiones impuestas por la cimentación de Rueda, por lo que aumentó la solidez de los pilares y el grueso de los muros. De forma inmediata, las autoridades aceptaron lo proyectado por Chacón, y las obras se iniciaron con la colocación de la primera piedra el 8 de diciembre de 1766 , coincidiendo con la celebración de las festividades en honor a la Inmaculada Concepción, patrona de la ciudad y protectora de la nueva catedral (E. Restrepo 239).

Fue el obispo Arauz quien el 5 de marzo de 1767 remitió a la Corte el proyecto de Chacón, acompañado de un nuevo plano que el ingeniero había firmado y fechado dos días antes del envío (figura 4). Este dibujo, considerado un testimonio gráfico fiable y correspondiente en gran medida a lo finalmente construido, ha servido tradicionalmente para fechar el proyecto. El plano se divide en tres partes bien diferenciadas (AGI, $S F$, sign. II88, "Carta del obispo de Santa Marta al gobernador", 5 de marzo de 1767). En la zona inferior, el ingeniero representó la planta de la nueva catedral, que parece seguir el modelo propuesto por Rueda. Ello hace pensar en la posibilidad de que Chacón reaprovechase la vieja cimentación, adaptando su nuevo edificio a las dimensiones del anterior. No obstante, incluyó ciertas novedades hasta entonces insólitas. Entre otras, introdujo un nuevo tramo a los pies dividido por pilares cruciformes que sustentarían un coro alto, a diferencia del concebido por Rueda en el centro 
de la nave. En cada uno de los laterales del coro se hallan el baptisterio y unas escaleras helicoidales para subir a las torres de la fachada. Precisamente acerca de las torres cabe llamar la atención sobre el interesante remate en forma de cúpula bulbosa, sin antecedentes en el panorama arquitectónico colombiano y que Corradine (222) ya relacionó con diferentes modelos propios de la arquitectura centroeuropea. Es más, este proyecto se debe valorar en tanto que constituye uno de los primeros ejemplos de fachada para catedral con dos torres integradas, pues si bien es cierto que en Monguí o en la iglesia de San Pedro Claver de Cartagena ya existían diseños parecidos, fue a partir de este momento que se difundió por el resto del virreinato.

En cuanto a la cabecera, la propuesta por Chacón y finalmente construida destaca por su complejidad, pues concibió una cripta bajo el altar para enterrar a los obispos y un corredor en la parte trasera como acceso a las viviendas de los sacristanes y a la sala capitular, estancias situadas a cada uno de los lados de la capilla mayor. Por último, aprovechando el espacio resultante en la parte trasera del altar y cerrando el testero de la catedral, incluyó un cementerio para el entierro de los canónigos.

El plano se completa con una sección del frente y del lateral de la catedral en la parte superior. En sendos dibujos Chacón mostró la cúpula que debía cerrar el crucero, concebida sobre un tambor circular y rematada por una linterna, de acuerdo con los postulados clasicistas. La inserción de una cúpula en el crucero ha sido relacionada con el modelo altamente divulgado en América por influencia de la Compañía de Jesús, como demuestra el caso de la iglesia de San Ignacio de Bogotá (Corradine 222). Además, se puede comprobar cómo la nave central es de mayor altura que las laterales, lo que permitió la inclusión de unas claraboyas circulares que iluminaban los tramos centrales de la iglesia. Igualmente novedosa en los contextos neogranadinos resultó la bóveda de cuarto de esfera ideada para el cierre de la cabecera, adaptando una solución ya manejada por los arquitectos italianos del Renacimiento. Entre otros, solución similar dispuso Andrea Palladio para los brazos de sus iglesias venecianas. Lo anterior demuestra un conocimiento por parte de Chacón de los repertorios constructivos recogidos en la literatura artística. Asimismo, la combinación de arcos de medio punto, pilastras toscanas dividiendo los tramos de la fachada y la escasez de decoración ahondan en el carácter clasicista del conjunto. En este sentido, las normas constructivas conocidas por el ingeniero durante su periodo de formación en la Academia de Matemáticas de Barcelona condicionaron el diseño de la nueva catedral. Durante el segundo curso del plan de estudios de 


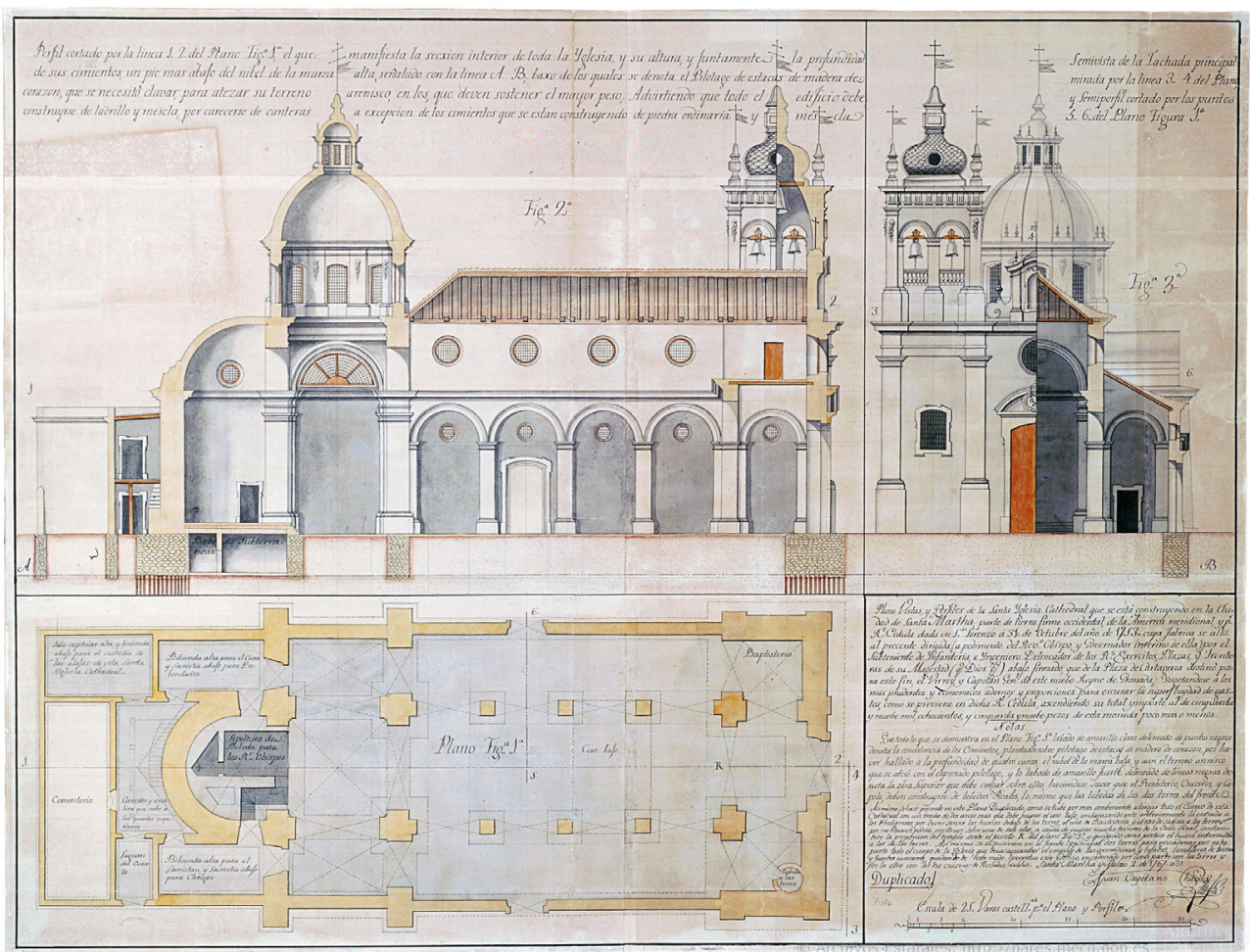

$\leftrightarrow$ FIGURA 4 .

Juan Cayetano Chacón. Proyecto para la catedral de Santa Marta, I767

Fuente: AGI, MP, P, I76.

dicha institución, los tratados de arquitectura renacentistas constituían las principales fuentes de aprendizaje para los cadetes, quienes aplicarían los modelos en sus diseños. Así, primero la Regla de Vignola y después el Compendio del padre Tosca incluían distintas anotaciones sobre las proporciones y medidas que encauzaron a los ingenieros hacia unos cánones clasicistas (Carrillo de Albornoz IOI-II6). Por todo ello, es posible que Chacón conociese estos diseños por medio de estampas, lo que habría influido en su propuesta del alzado exterior de la catedral samaria, en la que se apartó de la sobriedad y pobreza constructiva de otros edificios coetáneos.

De este modo, con el envío del plano y del expediente, el obispo Arauz buscaba obtener la financiación necesaria para construir tan ambicioso edificio, pues el pago de los gastos era inviable para las autoridades eclesiásticas locales. Ejercía entonces como gobernador y capitán comandante de la plaza Andrés Pérez Ruiz Calderón, con quien el obispo debía tratar acerca de los 
pagos, como responsable que era de administrar los fondos provistos por el virrey. Precisamente la obtención del dinero solicitado fue el principal escollo que debieron sortear tanto la gobernación como el propio Chacón, quien en mayo de 1767, ante la escasez de fondos, llegó a solicitar al virrey Messía de la Cerda su reincorporación a las obras de Cartagena, adonde había sido destinado como miembro del Real Cuerpo de Ingenieros (AGI, $S F$, sign. II88, "Carta del ingeniero Juan Cayetano Chacón”, 2 de mayo de 1767). Llama la atención el interés de Chacón por volver a trabajar en las fortificaciones cartageneras, pues concebía la construcción de la catedral como un proyecto que debía dirigir un arquitecto exento de encargos militares o ingenieriles.

Ante la amenaza de abandono de Chacón, tanto el nuevo obispo de la diócesis Manuel Camacho y Rojas como el gobernador Manuel de Herrera Leyba le exigieron una mayor presencia en la dirección de las obras y le solicitaron un informe en el que detallase el avance de la nueva construcción. El ingeniero firmó un detallado resumen del estado en que se encontraban las obras de la catedral el is de junio de 1767 . En su escrito apuntó que ya se había concluido la colocación del pilotaje de estacas de madera que servirían para atesar el terreno arenisco y fangoso. Sobre esta base se instalarían los cimientos, que ya se habían macizado hasta la superficie con mampuestos, grava y cemento. Ello había permitido iniciar el arranque de los pilares de la cripta proyectada para la cabecera, los del crucero izquierdo y los estribos exteriores. En lo que se refiere a la fachada, aún se estaban terminando las labores de cimentación de la primera torre, pues el peso de esta requería un mayor sustento (AGI, $S F$, sign. I188, “Carta de Juan Cayetano Chacón”, I5 de junio de 1767).

Por otro lado, dada la premura que existía por acabar el proyecto, el ingeniero informó de la construcción de un horno de cal en forma de campana de mediana proporción, con el fin de abaratar los costos que suponían la compra de este producto. Aunque no conservado en la actualidad, el horno debía coincidir con los fabricados en Cartagena de Indias durante las primeras décadas del siglo Xvin en el entorno de Tierra Bomba. El ingeniero pretendía con esta medida eliminar los excesivos gastos que ralentizaban la construcción, favoreciendo así el interés de unas autoridades que reclamaban de forma continua un mayor ajuste a los presupuestos iniciales. Es más, ante la falta de fondos, Camacho y Herrera reclamaron a la Corte nuevos pagos que permitiesen cerrar la cimentación e iniciar la elevación de los muros perimetrales de la nueva catedral.

Sin embargo, los continuos intentos por conseguir la financiación necesaria fueron en balde, pues las autoridades no hicieron caso a las constantes 
reclamaciones escritas por el gobernador Herrera y por el ingeniero Chacón. Es más, el 3 de junio de 1768 el propio militar reconoció que se habían acabado los fondos reservados para la obra después de iniciarse la elevación de los pilares torales, la nave principal y la cabecera. Ante el aparente abandono del proyecto, Chacón solicitó al virrey Messía de la Cerda su vuelta a las obras de Cartagena de Indias, pues reconocía que llevaba algún tiempo sin poder trabajar en Santa Marta (AGNC, MM, leg. I26, ff. 967-968). Fue en este momento cuando el ingeniero abandonó definitivamente las obras, dejando el proyecto inconcluso pero asentando las bases del que sería el edificio más representativo de la arquitectura neogranadina de las últimas décadas del siglo XVIII.

\section{La culminación del proceso constructivo}

Parece evidente que la falta de financiación y el regreso del ingeniero Juan Cayetano Chacón a las obras de Cartagena dificultaron la finalización de la nueva catedral de Santa Marta. De hecho, en I774 se detuvo definitivamente el pago de las herramientas y materiales, circunstancia que obligó a revocar una serie de decisiones en relación con el proyecto definitivo de la catedral. Por ello, no será hasta la intervención del ingeniero Antonio de Narváez y la Torre en agosto de 1778 cuando el proyecto obtenga el impulso definitivo. Narváez, que había sido destinado a Santa Marta dos años antes tras haber trabajado en las fortificaciones de Cartagena, se ocuparía de conseguir la financiación necesaria, realizando al efecto un nuevo plano de la catedral (figura 5). En este nuevo boceto, el ingeniero planteó la posibilidad de eliminar el primer tramo de naves situado a los pies del templo. Además, teniendo en cuenta la debilidad de la cimentación, descartó la construcción de una segunda torre, hecho que explica que finalmente solo se realizase la que hoy se conserva (figura 6). En lo referente a la cabecera, Narváez prescindió de las estancias que Chacón había ideado en ese entorno para diseñar un espacio más diáfano y utilitario. Sin duda, el diseño propuesto por este ingeniero condicionó la finalización de la catedral.

El impulso generado por la aparición del ingeniero Antonio de Narváez, quien llegó a ser gobernador de Santa Marta, permitió que en 1785 se nombrase como director de la obra al arquitecto Antonio Marchante. Con este definitivo nombramiento se pretendía dar por concluida la catedral tras un proceso constructivo tedioso. Marchante se encontraba un edificio con una planta definida, pero con unos alzados sin finalizar. 


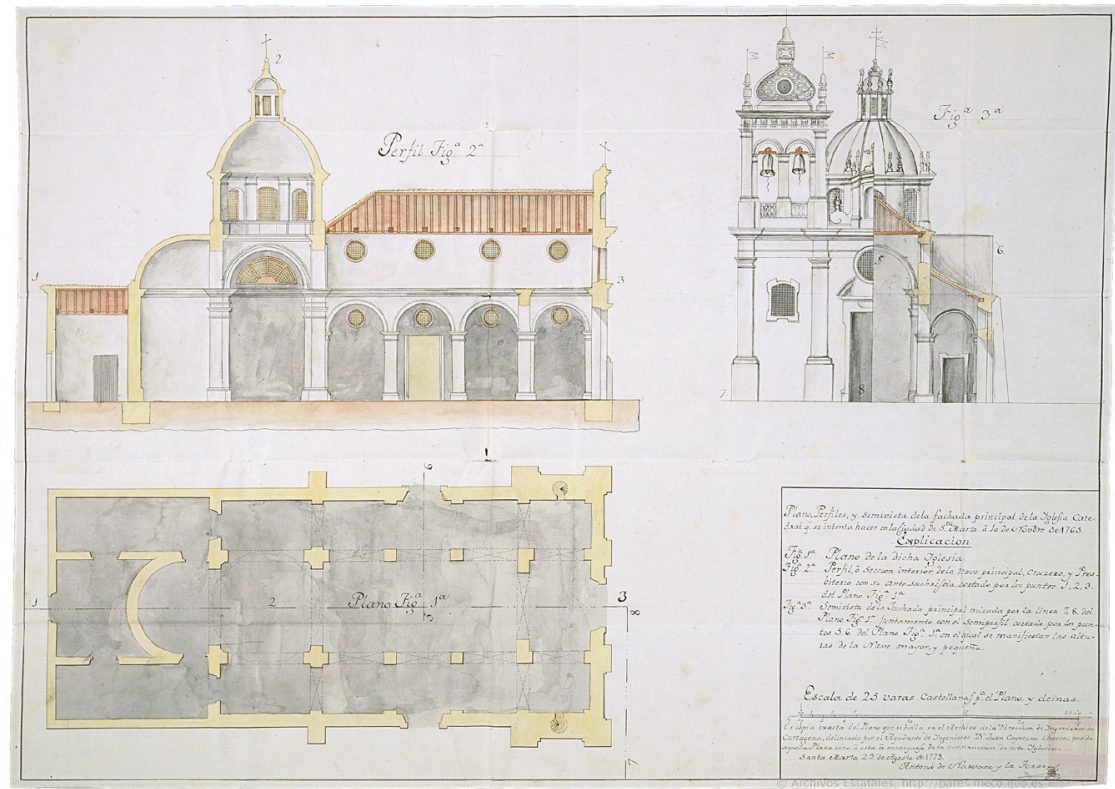

$\rightarrow$ FIGURA 5 .

Antonio de Narváez. Proyecto para la catedral de Santa Marta, I778 Fuente: AGI, MP, P, 256.

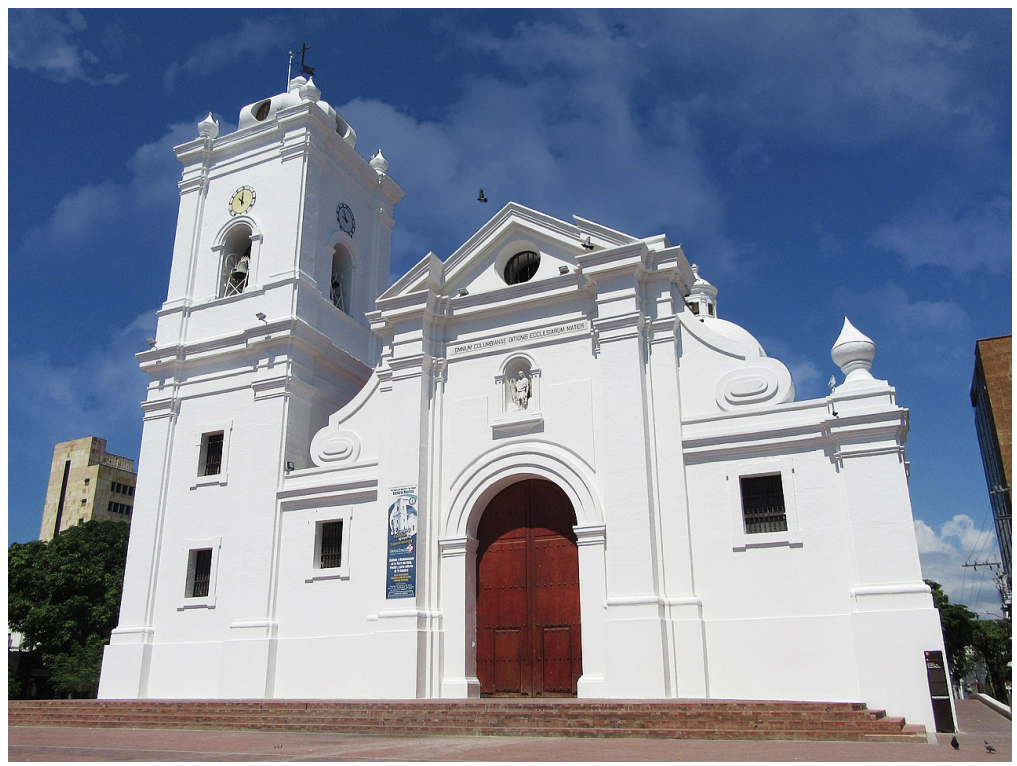

$\leftrightarrow$ FIGURA 6 .

Vista actual de la Catedral de Santa Marta, Colombia

Fuente: fotografía del autor.

$\begin{array}{llllllllllllllllllll}\mathbf{F} & \mathbf{R} & \mathbf{O} & \mathbf{N} & \mathbf{T} & \mathbf{E} & \mathbf{R} & \mathbf{A} & \mathbf{S} & d e & \text { la } & \text { H } & \mathbf{I} & \mathbf{S} & \mathbf{T} & \mathbf{O} & \mathbf{R} & \mathbf{I} & \mathbf{A}\end{array}$ 
Se debe por tanto a este periodo la construcción de la portada principal, en la que se insertaron una serie de elementos que difieren de lo proyectado por Narváez. Además de las pilastras gigantes que flanquean la portada principal, los aletones que sirven de tránsito entre las distintas naves aportan una monumentalidad distinta a lo concebido por los ingenieros, pues ambos elementos no aparecen representados en los planos ni descritos en los informes.

Como se ha apuntado, la falta de mano de obra local afectó a la terminación de la catedral. La sociedad indígena que habitaba de forma mayoritaria en la provincia samaria influyó en la ralentización del proyecto, al ser escasa la mano de obra capacitada. A ello habría que sumarle el papel desempeñado por la encomienda aún a fines del siglo xviII, circunstancia que derivó en un empobrecimiento poblacional y perjudicó notablemente la actividad arquitectónica en la ciudad.

Con todo ello, la catedral de Santa Marta debe ser valorada como un edificio fundamental para la confirmación de las tendencias clasicistas en el Virreinato del Nuevo Reino de Granada. Su disposición planimétrica, el desarrollo de sus alzados y su escasez decorativa obedecen a una concepción particular de la arquitectura propia de los ingenieros militares. Formados en la tradición impuesta por las academias de matemáticas, consideraban fundamental para un correcto diseño la utilización de las normas recibidas de la tradición clásica. Es en la transferencia de estos diseños desde la Corte al Nuevo Reino de Granada, mediante dibujos, estampas y modelos, donde reside la trascendencia de sus labores, siendo la catedral de Santa Marta el resultado de la vinculación indisoluble que existió entre los ingenieros militares y la arquitectura academicista en el Nuevo Mundo. La catedral ofrece una interesante síntesis de tendencias artísticas surgidas durante el siglo XvIII, reconociéndose como un ejemplo capital de las soluciones estructurales que se desarrollarían a lo largo de las siguientes centurias.

Por último, es de obligado cumplimiento llamar la atención sobre la importancia de esta catedral dentro de las políticas de evangelización implantadas por los Borbones. La resistencia mostrada por las tribus indígenas al supuesto dominio hispano obligó a los gobernadores a financiar un edificio que representase al estamento religioso ante la sociedad local. Por esta razón, la catedral de Santa Marta debe ser considerada la proyección arquitectónica de la Corona, imagen del poder ante las tribus sublevadas y representación de la autoridad impuesta contra los contrabandistas y las potencias enemigas presentes en el mar Caribe. 


\section{$\infty$ \\ B I B I L I O G R A F í A}

\section{F U E N T ES PR I MARIAS}

\section{A. Archivos}

Archivo General de Indias, Sevilla, España (AGI).

Mapas y Planos $(M P)$.

Panamá (P): 68, 175, 176, 256.

Santafé $(S F)$

Signatura Ir 88. Cartas y expedientes de Santa Marta

“Carta del obispo de Santa Marta José Javier Arauz”, is de abril de 1750.

"Carta del gobernador Antonio de Alcalá”, I4 de octubre de 1752.

"Carta del arquitecto Diego de Rueda", 5 de marzo de 1757.

"Carta del obispo de Santa Marta al gobernador", I3 de marzo de 1757.

"Carta del virrey Pedro Messía de la Cerda”, I2 de septiembre de 1766.

"Real Decreto", 5 de octubre de 1766.

"Carta del obispo de Santa Marta al gobernador", 5 de marzo de 1767.

"Carta del ingeniero Juan Cayetano Chacón”, 2 de mayo de 1767.

"Carta de Juan Cayetano Chacón”, is de junio de 1767.

Archivo General de la Nación, Bogotá, Colombia (AGNC).

Milicias y Marina (MM): leg. II7, I22, I26.

\section{FUENTESSECUNDARIAS}

Angulo Íñiguez, Diego. Planos de monumentos arquitectónicos de América y Filipinas existentes en el Archivo de Indias. Sevilla: Editorial Universidad, I939.

Arango Cardinal, Silvia. Historia de la arquitectura en Colombia. Bogotá: Universidad Nacional, 1993.

Bell Lemus, Carlos, coordinador. El Caribe colombiano. Guia de arquitectura y paisaje. Barranquilla-Sevilla: Junta de Andalucía, 2017.

Bermúdez, Arturo. Don Rodrigo de Bastidas: adelantado de Santa Marta. Bogotá: Fondo de Promoción a la Cultura, 2000.

---. Materiales para la historia de Santa Marta. Bogotá: Banco Central, I98I.

$\begin{array}{llllllllllllllllllll}\text { F } & \mathbf{R} & \mathbf{O} & \mathbf{N} & \mathbf{T} & \mathbf{E} & \mathbf{R} & \mathbf{A} & \mathbf{S} & d e & \text { la } & \text { H } & \mathbf{I} & \mathbf{S} & \mathbf{T} & \mathbf{O} & \mathbf{R} & \mathbf{I} & \mathbf{A}\end{array}$ 
Borrego Plá, Carmen. "Santa Marta a finales del siglo Xviıi: de la nada al ser”. Santa Marta del olvido al recuerdo. Historia económica y social de cuatro siglos, editado por Jorge Enrique Elías Caro. Santa Marta: Universidad del Magdalena, 20II, pp. 63-88. https:// doi.org/10.2307/j.cttızkomgv.7.

Capel Sáez, Horacio, et al. Los ingenieros militares en España. Siglo XVIII. Repertorio biográfico e inventario de su labor científica y espacial. Barcelona: Editorial Universidad, 1983.

Carrillo de Albornoz y Galbeño, Juan. "Los planes de estudios de la Academia de Matemáticas y su funcionamiento interno". La Academia de Matemáticas de Barcelona, editado por Juan Miguel Muñoz Corbalán. Barcelona: Ministerio de Defensa, 2004, pp. Ior-II6.

Castillero Calvo, Alfredo. Portobelo y el San Lorenzo del Chagre. Perspectivas imperiales. Siglos XVI-XIX. Tomo II. Panamá: Novo Art, 2016.

Castillo Oreja, Miguel Ángel y Mónica Riaza de los Mozos. "Entre el Barroco y el neoclasicismo: la Academia de Bellas Artes de San Fernando y las últimas empresas constructivas de los Borbones en América”. Actas III Congreso Internacional del Barroco Americano: Territorio, Arte, Espacio y Sociedad. Sevilla: Universidad Pablo de Olavide, 2001, pp. 708-724.

Corradine, Alberto. Historia de la arquitectura colombiana. 1538-1850. Bogotá: Biblioteca de Cundinamarca, 1989.

D'Amato Castillo, Guissepe. "Sobre arquitectos, planos y finanzas de la construcción de la catedral de Santa Marta a finales del siglo xviri”. Memorias. Revista Digital de Historia y Arqueología desde el Caribe, n. ${ }^{0}$ 27, 2015, pp. I-I6.

Galindo Díaz, Jorge. "El legado técnico de los tratados de fortificación en América hispánica”. Apuntes, vol. 17, n. ${ }^{\circ} \mathrm{I}-2,2004$, pp. 8-29.

Gámez Casado, Manuel. "De ingeniero voluntario a ingeniero extraordinario. El acceso al cuerpo de don Antonio de Narváez y la Torre". La formación artística: creadoreshistoriadores-espectadores, editado por Begoña Alonso et al. Santander: Universidad de Cantabria, 2018, pp. 237-246. https://doi.org/10.22429/Euc2018.062.

---. "Ingenieros militares y obras públicas. Algunos ejemplos de Nueva Granada durante el siglo XVIII". Ars Longa, n. ${ }^{\circ}$ 27, 2018, pp. I25-I38. https://doi.org/10.7203/arslonga.27.11019.

---. "La pacificación de La Guajira por el ingeniero Antonio de Arévalo. Sobre el proyecto de defensa de Sabana del Valle". Laboratorio de Arte, n. ${ }^{\circ}$ 28, 2016, pp. 373-386. https:// doi.org/10.12795/la.2006.i.01.20.

Hernández Ospino, William José. Historia de la Catedral de Santa Marta. Última morada de Simón Bolivar. Santa Marta: Universidad del Magdalena, 2003.

Marchena, Juan. "La primera Academia de Ingenieros en América”. Ejército. Revista de las Armas y Servicios, n. ${ }^{\circ} 447$, 1977, pp. 23-28. 
Marco Dorta, Enrique. "La arquitectura del siglo xviı en Panamá y en Colombia”. Historia del arte hispanoamericano, tomo I, editado por Diego Angulo Iñiguez. Madrid: Salvat, 1956, pp. 223-271.

Molino García, María Teresa. La encomienda en el Nuevo Reino de Granada durante el siglo XVIII. Sevilla: Consejo Superior de Investigaciones Científicas, 1976.

Navarro García, Luis. Hispanoamérica en el siglo XVIII. Sevilla: Editorial Universidad, I975.

Restrepo Olano, Margarita. Nueva Granada en tiempos del virrey Solís. 1753-176r. Bogotá: Universidad del Rosario, 2009.

Restrepo Tirado, Ernesto. Historia de la provincia de Santa Marta. Sevilla: Eulogio de Heras, 1929.

Ruiz Carrasco, Jesús María. "Los ingenieros militares y la censura de la Real Academia de Bellas Artes de San Fernando". Un mar fortificado: la defensa del caribe en el siglo XVIII $y$ sus antecedentes (en prensa).

Torres Lanzas, Pedro. Relación descriptiva de los mapas, planos de las antiguas audiencias de Panamá, Santa Fe y Quito existentes en el Archivo General de Indias. Madrid: Revistas de Archivos, Bibliotecas y Museos, 1904.

Zambrano, Fabio y Olivier Bernard. Ciudad y territorio: el proceso de poblamiento de Colombia. Bogotá: Academia de la Historia, 2014.

Zapatero, Juan Manuel. Historia de las fortalezas de Santa Marta y estudio asesor para su restauración. Bogotá: Academia de la Historia, 1980. 